\title{
Modelo de gestión por resultados para mejorar la calidad de gasto en la Unidad de Gestión Educativa Local, San Martín
}

\author{
Irvin Edilberto Guerra Rodríguez \\ chillosisa@gmail.com \\ Universidad César Vallejo \\ Gabriela del Pilar Palomino Alvarado \\ dpalominoal@ucvvirtual.edu.pe \\ Universidad César Vallejo
}

\section{RESUMEN}

El presente estudio tuvo como objetivo proponer y validar un modelo de gestión por resultados para mejorar la calidad de gasto en la Unidad de Gestión Educativa Local, con un enfoque cuantitativo de tipo aplicada, diseño no experimental descriptivo propositivo, presentó una población de 30 especialistas, siendo estos la muestra por el reducido tamaño, se aplicó como instrumento un cuestionario para la obtención de datos, es así que se obtuvo como resultado de acuerdo al criterio de los especialistas de la UGEL y directores de las instituciones educativas confirmaron la presencia de acciones inadecuadas inherentes a la calidad de gasto, identificándose entre ellas, el incumplimiento en la ejecución total del presupuesto asignado a la UGEL y como consecuencia los directores sufren limitantes en el desempeño de sus objetivos institucionales, de manera que las metas alcanzadas son insatisfactorias por los mismos, frente a esta realidad el desarrollo de modelo de gestión resulta importante y adecuada para la mejora considerable a nivel de procesos institucionales.

Palabras clave: gestión por resultados, calidad de gasto, modelo de gestión 


\title{
Results-based management model to improve the quality of spending in the Local Educational Management Unit, San Martín
}

\begin{abstract}
:
The objective of this study was to propose an validation a results-based management model to improve the quality of spending in the Local Educational Management Unit, with a quantitative approach of an applied type, non-experimental descriptive-purpose design, presented a population of 30 specialists, these being Due to the small size of the sample, a questionnaire was applied as an instrument to obtain data, so it was obtained as a result according to the criteria of UGEL specialists and directors of educational institutions confirmed the presence of inappropriate actions inherent to the quality of spending, identifying among them the non-compliance in the total execution of the budget assigned to the UGEL and as a consequence the directors suffer limitations in the performance of their institutional objectives, so that the goals achieved are unsatisfactory by them, compared to this reality the development of the management model is important and appropriate for considerable improvement at the level of institutional processes.
\end{abstract}

Keywords: management by results, quality of spending, management model

Artículo recibido: 02 oct. 2020 Aceptado para publicación: 14 nov. 2020 Correspondencia: chillosisa@gmail.com. Conflictos de Interés: Ninguna que declarar 


\section{INTRODUCCIÓN}

A nivel internacional se reconoce que la calidad de gasto está referida a aquellas acciones en las que se incurrieron gastos públicos de modo eficiente, para lo cual es sumamente indispensable que se cumplan ciertos lineamientos y normativas que buscan contribuir con el crecimiento y desarrollo de nuestro país y, a su vez, cubrir las necesidades y requerimientos de la ciudadanía. En ese sentido, es propicio afirmar que existirá una eficiente calidad de gasto cuando los responsables planteen objetivos claros y tomen decisiones asertivas de manera participativa y razonable dentro de la planificación estratégica. Al respecto, Torres (2017) sostiene que ello contiene una serie de componentes que contribuyen con la utilización efectiva de los medios con el fin de contribuir significativamente con el crecimiento económico de la nación y garantizar el progreso equitativo y distribuido a toda la población, por lo que es sumamente indispensable para garantizar una gestión pública transparente. Galiya et al. (2018) manifiestan que el presupuesto público está elaborado en función a la capacidad de ingreso y gasto de las entidades e instituciones estatales, por cuanto en base a ello puede medirse la eficiencia de las mismas; cabe mencionar que todas las entidades estatales y organismos gubernamentales disponen de un presupuesto asignado para ejecutar sus gastos, los mismos que deben estar consignados para la mejorara de sus infraestructuras, salud pública, seguridad pública, etc. No obstante, por medio de los resultados de un censo sectorial se ha podido reconocer que la mayoría de las instituciones públicas de los países Latinoamericanos perciben que existen numerosas falencias significativas concernientes al cumplimiento de las normativas, las mismas que logran afectar de manera directa los resultados programados, pues los responsables de estas instituciones no logran enfocar sus recursos en la mejora de la implementación de políticas, imposibilitando que se cumplan las metas planificadas (UNESCO, 2017).

En el Perú se reconoce que el Ministerio de Economía y Finanzas (MEF) (2016) por medio del Presupuesto por Resultados - PpR busca asignar sus recursos progresivamente hacia las instituciones y organismos estatales con el fin de garantizar que se cumplan sus metas institucionales previamente programadas y priorizadas en función a las necesidades de la población; en concordancia a ello, es propicio mencionar que este presupuesto asume una de las funciones indispensables para la distribución de los recursos públicos por medio del presupuesto, por cuanto realiza una evaluación previa de los requerimientos de cada Unidad 
Ejecutora con el propósito de asegurar la calidad de gasto público de las entidades, y además evalúa el efecto que ha tenido en el desarrollo de las mismas, buscando conocer los beneficios que han producido dentro de un ámbito específico. Así pues, Silva y Mejía (2017) aluden que los gestores públicos deben encontrase debidamente comprometidos con el desarrollo de una gestión pública eficiente enfocada al cumplimiento de las normativas de modo que se pueda garantizar la obtención de resultados positivos, los mismos que deben estar direccionados principalmente en beneficio de la población estudiantil, debido a que se considera que el desarrollo del sector educación debe ser esencial para asegurar el progreso de un país a través de la creación de ciudadanos debidamente preparados personal e intelectualmente.

En un contexto local, se presenta a la Unidad de Gestión Educativa Local San Martín, la cual es definida como una organización pública descentralizada cuya jurisdicción territorial de atención corresponde a la provincia de San Martín y propone como misión ofrecer un servicio educativo de calidad, asegurando la formación integra del cuerpo estudiantil y permitiendo la integración social concerniente a los cambios tecnológico-científicos que exige el mundo globalizado. Así pues, se percibe que dentro de la organización existen deficiencias relacionadas con el desarrollo de sus funciones que afectan su buen funcionamiento. Con respecto a la distribución de sus materiales, se reconoce que la organización no realiza el seguimiento preciso de cada uno de los procesos y lineamientos relacionados con la entrega de materiales educativos a las distintas instituciones educativas que pertenecen a su jurisdicción. De igual manera, se evidencia que los materiales distribuidos (materiales educativos y fungibles) presentan deterioros, más que todo en zonas rurales, llegando a ser recepcionados en muchas oportunidades casi obsoletos o con algún tipo de deficiencias, es decir, mojados, rotos, manchados con barro, debido a que las condiciones en las que se brindan los servicios de distribución no cumplen con los procedimientos para garantizar la integridad de estos materiales o estos no son exigidos al momento de elaborar las bases del proceso. Asimismo, en lo referente a la contratación de la plana docente, el cual involucra una cifra significativa en la ejecución del presupuesto anual de la UGEL, no se cuenta con los filtros necesarios para optimizar la contratación del docente de aula y que en muchas ocasiones estos son destacados a la UGEL para ocupar cargos de especialistas u otra función administrativa o de apoyo, toda vez que, en el proceso de contratación, que está regulado por normativa, no incluye las etapas de entrevista y/o 
examen de conocimiento y la verificación en línea de los títulos emitidos por las escuelas superiores de educación pedagógica; siendo estas parte fundamental en un proceso de selección de personal, con la finalidad de medir el grado de conocimiento o algunas aptitudes que estos deben cumplir, cabe precisa que, durante los últimos años se incrementaron los casos títulos falsos de docentes, es más, algunos de estos docentes ya estaban nombrados, ocasionando perjuicio a la entidad y al estado, asimismo, de igual manera, los presupuestos que se destinan para el mantenimiento de las instituciones educativas, que se realiza a través de encargos y con deposito directamente a la cuenta bancaria del director de la IE, estos presupuestos no son utilizados de manera eficaz y eficiente, es decir, no son ejecutados para los fines que estos fueron transferidos, obedeciendo muchas veces a gastos innecesarios y de índole personal; es más, existen directores que no rinden estos encargos, transgrediendo la normativa, por si fuera poco, estos directores siguen siendo contratados para ocupar cargos directivos, impidiendo de esta manera que se cumplan con la finalidad pública que tiene los presupuestos. En tal sentido, y frente a las deficiencias mencionadas con anterioridad, esta investigación pretende estudiar la realidad presentada y plantear medidas correctivas que permitan mejorar el funcionamiento de la entidad. En ese sentido, de acuerdo a la realidad descrita se buscó como objetivo la propuesta y validación de un modelo de gestión por resultados para mejorar la calidad de gasto en la Unidad de Gestión Educativa Local, San Martín.

Un análisis de la realidad busca el análisis de diversos estudios que sustenten el proceso diagnóstico y propositivo del modelo, esto a nivel internacional se presentó la investigación de Medina \& Hernández (2017) donde concluye que las empresas chilenas no son conscientes al momento de rendir cuentas, asimismo los mecanismos y herramientas no son empleadas de manera adecuada puesto que el $65 \%$ de las entidades estatales no tienen conocimiento suficiente para manejar los procedimientos de control de sus gestiones, permitiendo conocer así las falencias que se presentan durante el seguimiento de los procedimientos. Chica (2017) mediante el análisis de la gestión, evidencia que la gobernanza es inadecuada en un $90 \%$, por cuanto la gestión pública no responde a los requerimientos de la población y las obras públicas programadas para el presente periodo quedaron pendientes para el próximo periodo; además las actividades no están enfocadas en reducir pobreza y demás aspectos que impiden el crecimiento sostenible del país. En tanto Bastidas \& Andocilla (2016) indican que el 56\% afirma que el nivel de calidad de 
gasto es regular, pues se presenta en función a la transparencia en el seguimiento de los procesos de ejecución presupuestal para cada una de las obras programadas. No obstante, se reconoce que los recursos y medios del Estado no están distribuidos efectiva y equitativamente, por tal motivo los gobiernos de países latinoamericanos deben exigir una buena calidad de gasto al momento de ejecutar obras públicas.

En un contexto nacional, Ríos (2018) demuestra que existe una relación significativa en un $55 \%$ entre la gestión por resultados y la calidad de gasto, por cuanto el coeficiente de determinación alcanzado fue 0.645 con un nivel de significancia inferior a 0.05 , pues la deficiente gestión por resultados suscita una baja calidad de gasto en la entidad. De igual manera Franciskovic (2016) la elaboración del Presupuesto por Resultados es considerado una herramienta de mejora de la gestión pública, sin embargo, se percibe que, pese a los numerosos esfuerzos de la distintas entidades y organismos gubernamentales, las fuentes de información son poco eficientes, dificultando así la medición de los resultados, rendición de cuentas e imposibilitando la participación activa de la población. Asimismo, Barrenechea (2018) indica que la relación de una variable con otra es significativa en un $62 \%$, por cuanto el coeficiente de correlación fue 0.789 , permitiendo aceptar la hipótesis alterna en la cual se afirma que una variable se relaciona significativamente con otra. Desde una perspectiva local, Gormas (2019) indica que una variable se relaciona de manera significativa con otra en un $61 \%$, por cuanto el coeficiente obtuvo un 0.78 , facilitando la refutación de la hipótesis nula que niega que las variables guardan relación una con otra. Por otra parte, Vargas (2018). Concluye que se reconoce que la relación que existe entre una variable con otra es directa y positiva en un $73 \%$, por cuanto el coeficiente determinante fue 0.857 cuyo nivel de significancia fue 0,001 , pues se evidencia que el deficiente seguimiento de las acciones administrativas produce efectos negativos en la gestión por resultados, imposibilitando el crecimiento económico de la localidad. Dávila (2019), menciona que existe una relación directa entre ambas variables en un $27 \%$, puesto que el coeficiente determinante fue 0.518 , pues una gestión por resultados eficaz permite la adecuada programación y asignación de los recursos del Estado a través de los presupuestos elaborados en función a las necesidades de la entidad.

Desde el abordaje teórico fue necesario la definición conceptual de los mismos, iniciando por calidad de gasto, García (2015) sostiene que representa el nivel de utilización óptima de los recursos proporcionados por el Estado en la realización de obras y prestación de 
servicios públicos a favor de la ciudadanía con la finalidad de optimizar el nivel de vida. Refleja el grado de efectividad en el uso equitativo, sostenible y transparente de los recursos públicos, asimismo implica una serie de procesos por medio de los cuales se asegura la efectividad de los mismos (Álvarez \& Polo, 2018; Botero \& García, 2018). Además, es considerado como un mecanismo a través del cual se puede reducir los índices de pobreza, contribuir con el movimiento económico y consolidar el desarrollo del país de manera sostenida (Campos \& Mendoza, 2018; Fonseca \& López, 2018). Finalmente, la calidad del gasto está enfocada generalmente en atender las necesidades de la ciudadanía y no, como usualmente se reconoce, las necesidades de las entidades estatales, es decir, busca conocer en qué medida se proporcionan beneficios a los pobladores (Levy, 2016).

En cuanto a la gestión por resultados el MEF (2018) sostiene que es el procedimiento mediante el cual se administran los recursos públicos, los mismos que deben estar destinados al desarrollo de las actividades y operaciones establecidas en los planes gubernamentales que deben ser cumplidos dentro de un periodo determinado específico. Además, representa un mecanismo que permite controlar el aumento de los gastos, pues está explícitamente vinculado con el aumento de la capacidad productiva de bienes, servicios y resultados, asimismo, requiere de la constante evaluación de los resultados alcanzados en función a los objetivos propuestos (Ramos, 2016; Bermeo et al., 2018).

En última instancia la evolución histórica de los modelos de gestión, a lo largo de las últimas décadas, suscitaron una serie de acontecimientos que afectaron significativamente el desempeño de las entidades e instituciones estatales de diversos países, por lo que los ciudadanos vieron la necesidad de exigir una eficiente prestación de servicios públicos para optimizar el nivel de vida de la población en función a los indicadores de educación, salud, seguridad, etc. En tal sentido, la creación y diseño de un modelo de gestión implica una serie de procedimientos de planificación y gestión enfocados en objetivos concretos, incluyendo además indicadores que permitan evaluar el rendimiento del personal y las áreas operativas de la organización en base a los resultados obtenidos (Soberanes et al., 2018; Ferrer, 2018)

\section{ESTRATEGIAS METODOLÓGICAS O MATERIALES Y MÉTODOS}

El estudio presentó un enfoque cuantitativo, de tipo Aplicada CONCYTEC (2018), fundamenta que el objetivo principal es la solución de problemas mediante la aplicación de conocimientos e investigación que faciliten su desarrollo, con diseño no experimental, se 
ha fundamentado a través de la observación de los fenómenos tal y como se presentan, para ser analizados posteriormente (Bernal, 2010).

Se presenta un estudio descriptivo-transversal-propositivo, dado que se ha detallado información relacionadas a las variables en estudio, deseando brindar un mejor entendimiento; asimismo, fue propositivo porque estuvo constituido por una propuesta de un modelo de gestión por resultados con el fin de optimizar la calidad de gasto y finalmente fue correlacional, pues en esta investigación se ha determinado la relación que existe entre una variable y otra (Abanto, 2014)

Se contó como población a 30 especialistas pedagógicos de la Unidad de Gestión Educativa Local, San Martín y 311 directores de las instituciones educativas a nivel regional, siendo esto la totalidad de la muestra por el reducido número, además se tuvo a 54 directores, se consideró hacer uso de la muestra pirobalística, de tipo aleatorio simple, Arbaiza (2019).

Se ha utilizado como técnica de acuerdo a Arbaiza (2019) la encuesta, la misma que consiste en captar información relacionado a las variables, para la muestra se consideró lo mencionado por Cantillo y Buitrago (2018) haciendo uso del cuestionario, dado que estuvo conformado por medio de preguntas, teniendo en cuenta dimensiones e indicaciones de las variables, con el propósito de recopilar información necesaria para el desarrollo de la investigación, estos contaron con confiabilidad y validez.

En cuanto a los procedimientos se inició por la fase de diagnóstica, en esta fase se ha realizado un diagnóstico de la situación actual de la gestión por resultados en la calidad de gasto en la Unidad de Gestión Educativa Local por medio de los instrumentos elaborados para cada variable, deseando identificar, analizar y describir los resultados posteriormente obtenidos, las mismas que nos ayudaron al cumplimiento de los objetivos propuestos; asimismo, se utilizado la prueba estadística SPSS 25, la cual se ha empleado un análisis cuantitativo, pues se representaran por tablas. Seguido la fase propositiva, se tuvo en consideración la fase propositiva porque se ha procedido a diseñar una propuesta de gestión por resultados para mejorar la calidad de gatos en la Unidad de Gestión Educativa Local, deseando disminuir las falencias evidenciadas, tal y como se detalla en la realidad problemática del estudio.

De acuerdo a los objetivos propuestos en el estudio, se ha aplicado un método descriptivo, por cuanto al obtener los resultados tras la aplicación de los instrumentos, fue necesario asignar un número a cada respuesta (codificación), luego se ha efectuado a la tabulación de 
los resultados, para ello fue necesario emplear Microsoft Excel para el procesamiento de los datos, las funciones empleados fueron las tablas dinámicas, fórmulas y figuras Excel, conteos, sumas, multiplicación, aleatorios, porcentajes y promedios, cuyos resultados fueron presentados en tablas y figuras, que permitirán una mejor presentación y entendimiento de los resultados y datos obtenidos con respecto a las variables. Asimismo, se ha empleado el software estadístico SPSS 25, para el procesamiento de los datos, con respecto al cálculo del alfa de cronbach.

\section{RESULTADOS Y DISCUSIÓN}

Caracterizar el estado actual de la calidad de gasto en la Unidad de Gestión

\section{Educativa Local, San Martín}

\section{Tabla 1}

Calidad de gasto desde la percepción de los especialistas de la UGEL

\begin{tabular}{|c|c|c|c|c|c|c|c|}
\hline \multirow{2}{*}{\multicolumn{2}{|c|}{ Indicadores de calidad del gasto }} & \multicolumn{2}{|c|}{$\begin{array}{c}\text { Nunca / } \\
\text { Casi nunca }\end{array}$} & \multicolumn{2}{|c|}{ A veces } & \multicolumn{2}{|c|}{$\begin{array}{c}\text { Siempre / } \\
\text { casi } \\
\text { siempre }\end{array}$} \\
\hline & & $\mathbf{f i}$ & $\%$ & fi & $\%$ & fi & $\%$ \\
\hline \multirow{3}{*}{ Eficacia } & $\begin{array}{l}\text { El presupuesto asignado por la } \\
\text { UGEL, permite cumplir los } \\
\text { objetivos programados }\end{array}$ & 14 & $46.7 \%$ & 12 & $40.0 \%$ & 4 & $13.3 \%$ \\
\hline & $\begin{array}{l}\text { Las metas alcanzadas por la I.E. } \\
\text { son satisfactorias }\end{array}$ & 20 & $66.7 \%$ & 9 & $30.0 \%$ & 1 & $3.3 \%$ \\
\hline & $\begin{array}{l}\text { La UGEL ejecuta el total de su } \\
\text { presupuesto }\end{array}$ & 17 & $56.7 \%$ & 10 & $33.3 \%$ & 3 & $10.0 \%$ \\
\hline \multirow{3}{*}{ Eficiencia } & $\begin{array}{l}\text { La UGEL optimiza y controla el } \\
\text { uso del presupuesto asignado. }\end{array}$ & 11 & $36.7 \%$ & 14 & $46.7 \%$ & 5 & $16.7 \%$ \\
\hline & $\begin{array}{lrr}\text { La calidad de los materiales } \\
\text { educativos } & \text { satisface las }\end{array}$ & 10 & $33.3 \%$ & 12 & $40.0 \%$ & 8 & $26.7 \%$ \\
\hline & necesidades de las I.E & & & & & & \\
\hline \multirow{2}{*}{ Relevancia } & $\begin{array}{l}\text { La UGEL, ejecuta el presupuesto } \\
\text { considerando el cumplimiento de } \\
\text { los objetivos principales de las IE }\end{array}$ & 12 & $40.0 \%$ & 9 & $30.0 \%$ & 9 & $30.0 \%$ \\
\hline & $\begin{array}{l}\text { La UGEL, prioriza la realización } \\
\text { de actividades para el efectivo } \\
\text { aprendizaje del alumno }\end{array}$ & 6 & $20.0 \%$ & 10 & $33.3 \%$ & 14 & $\begin{array}{l}46.7 \\
\%\end{array}$ \\
\hline \multirow[b]{2}{*}{ Pertinencia } & 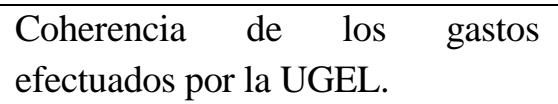 & 13 & $43.3 \%$ & 9 & $30.0 \%$ & 8 & $26.7 \%$ \\
\hline & $\begin{array}{l}\text { La UGEL, prioriza el presupuesto } \\
\text { para actividades educativas } \\
\text { orientadas a una educación } \\
\text { diversificada. }\end{array}$ & 4 & $13.3 \%$ & 11 & $36.7 \%$ & 15 & $\begin{array}{l}\mathbf{5 0 . 0} \\
\%\end{array}$ \\
\hline
\end{tabular}




\begin{tabular}{|c|c|c|c|c|c|c|c|}
\hline \multirow[b]{2}{*}{ Equidad } & $\begin{array}{l}\text { La distribución de los recursos } \\
\text { educativos, es justa y equitativo }\end{array}$ & 21 & $70.0 \%$ & 6 & $20.0 \%$ & 3 & $10.0 \%$ \\
\hline & $\begin{array}{l}\text { La UGEL distribuye el presupuesto } \\
\text { considerando a la población escolar } \\
\text { más vulnerable. }\end{array}$ & 5 & $16.7 \%$ & 9 & $30.0 \%$ & 16 & $\begin{array}{l}53.3 \\
\%\end{array}$ \\
\hline \multirow{2}{*}{ Transparencia } & $\begin{array}{l}\text { Fácil acceso a la información del } \\
\text { gasto educativo realizado por la } \\
\text { UGEL }\end{array}$ & 21 & $70.0 \%$ & 6 & $20.0 \%$ & 3 & $10.0 \%$ \\
\hline & $\begin{array}{l}\text { Proporciona la UGEL información } \\
\text { sobre los logros educativos } \\
\text { alcanzados. }\end{array}$ & 17 & $56.7 \%$ & 10 & $33.3 \%$ & 3 & $10.0 \%$ \\
\hline \multirow{2}{*}{$\begin{array}{l}\text { Sostenibilida } \\
\text { d }\end{array}$} & $\begin{array}{l}\text { La UGEL, cuenta con } \\
\text { disponibles parsos } \\
\text { necesidades de las IE. }\end{array}$ & 18 & $60.0 \%$ & 7 & $23.3 \%$ & 5 & $16.7 \%$ \\
\hline & $\begin{array}{l}\text { Suficiencia del presupuesto } \\
\text { designado por la UGEL, para } \\
\text { atender las necesidades de las I.E }\end{array}$ & 14 & $46.7 \%$ & 10 & $33.3 \%$ & 6 & $20.0 \%$ \\
\hline
\end{tabular}

Fuente: Cuestionario aplicado a los especialistas de la UGEL

Del 100\% (30) de los encuestados, referente a la dimensión eficacia, el 66.7\% (20) manifiestan que "Nunca o casi nunca" son satisfactorias las metas alcanzadas por las I.E.; asimismo, que la UGEL no ejecuta el total de su presupuesto (56,7\%), y lo asignado no permite cumplir con los objetivos programados (46.7\%).

En cuanto a la dimensión eficiencia, el 46.7\% (14) opinan que "A veces" la UGEL optimiza y controla el uso del presupuesto asignado para las acciones educativas y que la calidad de los materiales distribuidos satisface las necesidades (40.0\%) con tendencia a que dicha acción sea "Nunca o casi nunca". En tanto la dimensión Relevancia, el 46.7\% (14) aseguran que "Siempre o casi siempre" se priorizan la realización de las actividades para el efectivo aprendizaje de los alumnos, aunque los especialistas indican que "Nunca o casi nunca" la UGEL ejecuta el presupuesto considerando la necesidad de cumplir los objetivos de la I.E (40.0\%); de la misma manera en la dimensión Pertinencia, el 50\% (15) refieren que "Siempre o casi siempre" se prioriza el presupuesto para las actividades educativas orientadas a una educación diversificada, sin embargo, el 43.3\% (13) percibe que "Nunca o casi nunca" existe coherencia de los gastos efectuados por la UGEL. De acuerdo con la dimensión Equidad, el 70\% (21) sostienen que "Nunca o casi nunca" existe una distribución equitativa y justa de los recursos educativos a las I.E. Así también en la dimensión Transparencia, el 70\% (21) aluden que "Nunca o casi nunca" la comunidad 
educativa tiene un fácil acceso a información relacionada al gasto pùblico ejecutado por la UGEL, ni proporciona información sobre los logros educativos alcanzados (56.7\%). Finalmente, la dimensión Sostenibilidad, el 60\% (18) infieren que "Nunca o casi nunca" la UGEL cuenta con recursos disponibles para atender las necesidades de las I.E, ni el presupuesto designado por la UGEL, es suficiencia para atender las necesidades de las I.E $(46.7 \%)$.

\section{Tabla 2}

Calidad de gasto desde la percepción de los directores de las I.E

\begin{tabular}{|c|c|c|c|c|c|c|c|}
\hline \multicolumn{2}{|c|}{ Indicadores de calidad del gasto } & \multicolumn{2}{|c|}{$\begin{array}{c}\text { Nunca / Casi } \\
\text { nunca }\end{array}$} & \multicolumn{2}{|c|}{ A veces } & \multicolumn{2}{|c|}{$\begin{array}{c}\text { Siempre / casi } \\
\text { siempre }\end{array}$} \\
\hline & & fi & $\%$ & fi & $\%$ & fi & $\%$ \\
\hline \multirow{3}{*}{ Eficacia } & $\begin{array}{l}\text { El presupuesto } \\
\text { proporcionado por la UGEL, } \\
\text { permite cumplir los } \\
\text { objetivos programados en las } \\
\text { I.E }\end{array}$ & 35 & $64.8 \%$ & 17 & $31.5 \%$ & 2 & $3.7 \%$ \\
\hline & $\begin{array}{l}\text { Las metas alcanzadas por su } \\
\text { I.E. responden al } \\
\text { presupuesto asignado por la } \\
\text { UGEL }\end{array}$ & 24 & $44.4 \%$ & 26 & $48.1 \%$ & 4 & $7.4 \%$ \\
\hline & $\begin{array}{l}\text { La UGEL ejecuta el total de } \\
\text { su presupuesto asignado. }\end{array}$ & 37 & $68.5 \%$ & 14 & $25.9 \%$ & 3 & $5.6 \%$ \\
\hline \multirow{2}{*}{ Eficiencia } & $\begin{array}{l}\text { La UGEL optimiza y } \\
\text { controla el uso del } \\
\text { presupuesto asignado para } \\
\text { las acciones educativas }\end{array}$ & 25 & $46.3 \%$ & 24 & $44.4 \%$ & 5 & $9.3 \%$ \\
\hline & $\begin{array}{l}\text { La proporción y la calidad de } \\
\text { los materiales educativos } \\
\text { satisfacen las necesidades de } \\
\text { su I.E }\end{array}$ & 24 & $44.4 \%$ & 21 & $38.9 \%$ & 9 & $16.7 \%$ \\
\hline \multirow{2}{*}{ Relevancia } & $\begin{array}{l}\text { La UGEL, le asigna } \\
\text { presupuesto teniendo en } \\
\text { cuenta el cumplimiento de } \\
\text { los objetivos }\end{array}$ & 18 & $33.3 \%$ & 24 & $44.4 \%$ & 12 & $22.2 \%$ \\
\hline & $\begin{array}{l}\text { Priorización en la realización } \\
\text { de actividades para el } \\
\text { efectivo aprendizaje del } \\
\text { alumno }\end{array}$ & 12 & $22.2 \%$ & 8 & $14.8 \%$ & 34 & $63.0 \%$ \\
\hline Pertinencia & $\begin{array}{l}\text { Coherencia de los gastos } \\
\text { efectuados por la UGEL. }\end{array}$ & 20 & $37.0 \%$ & 21 & $38.9 \%$ & 13 & $24.1 \%$ \\
\hline
\end{tabular}




\begin{tabular}{|c|c|c|c|c|c|c|c|}
\hline & $\begin{array}{l}\text { La UGEL prioriza el } \\
\text { presupuesto para actividades } \\
\text { educativas orientadas a una } \\
\text { educación diversificada }\end{array}$ & 12 & $22.2 \%$ & 18 & $33.3 \%$ & 24 & $44.4 \%$ \\
\hline \multirow[b]{2}{*}{ Equidad } & $\begin{array}{l}\text { Equidad en la distribución de } \\
\text { los recursos educativos en su } \\
\text { I.E. }\end{array}$ & 23 & $42.6 \%$ & 19 & $35.2 \%$ & 12 & $22.2 \%$ \\
\hline & $\begin{array}{l}\text { La UGEL distribuye el } \\
\text { presupuesto considerando a } \\
\text { la población escolar más } \\
\text { vulnerable }\end{array}$ & 26 & $48.1 \%$ & 17 & $31.5 \%$ & 11 & $20.4 \%$ \\
\hline \multirow{2}{*}{ Transparencia } & $\begin{array}{l}\text { Fácil acceso a la información } \\
\text { del gasto educativo realizado } \\
\text { por la UGEL }\end{array}$ & 34 & $63.0 \%$ & 16 & $29.6 \%$ & 4 & $7.4 \%$ \\
\hline & $\begin{array}{l}\text { La UGEL proporciona la } \\
\text { información sobre los logros } \\
\text { educativos alcanzados }\end{array}$ & 27 & $\mathbf{5 0 . 0 \%}$ & 14 & $25.9 \%$ & 13 & $24.1 \%$ \\
\hline \multirow[b]{2}{*}{ Sostenibilidad } & $\begin{array}{l}\text { Recursos disponibles para } \\
\text { atender las necesidades de su } \\
\text { I.E. }\end{array}$ & 17 & $31.5 \%$ & 13 & $24.1 \%$ & 24 & $44.4 \%$ \\
\hline & $\begin{array}{l}\text { Suficiencia de presupuesto } \\
\text { designado por la UGEL, } \\
\text { para atender las necesidades } \\
\text { prioritarias de su I.E }\end{array}$ & 32 & $59.3 \%$ & 9 & $16.7 \%$ & 13 & $24.1 \%$ \\
\hline
\end{tabular}

Fuente: Cuestionario aplicado a los directos de las Instituciones educativas

Del 100\% (54) de los directores de las instituciones educativas, refieren que, en la dimensión eficacia, el 68.5\% (37) consideran que "nunca o casi nunca" la UGEL, ejecuta el total de su presupuesto asignado, ni el presupuesto proporcionado por la UGEL, les permite cumplir con la totalidad de sus objetivos institucionales (64.8\%), en tanto el $48.1 \%$ perciben que "a veces" las metas alcanzadas por su institución responden al presupuesto asignado por la UGEL. De la misma manera, en la eficiencia, el 46.3\% (25) revelan que "nunca o casi nunca" la UGEL, optimiza y controla el presupuesto designado para las acciones educativas, asimismo que la proporción y calidad de los materiales no satisface las necesidades de sus instituciones (44.44\%). Con respecto, a la Relevancia, el 63\% (34) aseguran que "siempre o casi siempre" se priorizan aquellas actividades que generan un afectivo aprendizaje de los alumnos, y a veces la UGEL, les asigna presupuesto teniendo en cuenta el cumplimiento de sus objetivos institucionales (44.4\%)

Así también, en la Pertinencia, el 44.4\% (24) refieren que "siempre o casi siempre" la UGEL, prioriza el presupuesto para aquellas actividades orientadas a una educación diversificada, pero, "A veces" existe coherencia de los gastos efectuado por la misma 
(38.9\%). De acuerdo a la Equidad, el 48.1\% (26) sostienen que "nunca o casi nunca" la UGEL distribuye el presupuesto considerando a la población escolar más vulnerable, además se percibe falta de equidad en la distribución de los recursos educativos (42.6\%), Así también en la Transparencia, el 63\% (34) aluden que "nunca o casi nunca" la comunidad educativa tiene fácil acceso a la información relacionada a los gastos efectuados por la UGEL. Por lo demás, el 50\% (27) asegura que la UGEL no suele proporcionar información sobre los logros alcanzados. Finalmente, la Sostenibilidad, el 59.3\%(32) infieren que "nunca o casi nunca" el presupuesto asignado a las instituciones es suficiente para atender sus necesidades institucionales, sin embargo, consideran que "siempre o casi siempre" existe recursos disponibles para atender sus necesidades institucionales (44.4\%).

Caracterizar el estado actual de la gestión por resultados en la Unidad de Gestión

\section{Educativa Local, San Martín}

Tabla 3

Gestión por resultados desde la percepción de los especialistas de la UGEL

\begin{tabular}{|c|c|c|c|c|c|c|c|}
\hline \multicolumn{2}{|c|}{ Indicadores de la gestión por resultados } & \multicolumn{2}{|c|}{$\begin{array}{c}\text { Nunca/Casi } \\
\text { nunca }\end{array}$} & \multicolumn{2}{|c|}{ A veces } & \multicolumn{2}{|c|}{$\begin{array}{c}\text { Siempre/casi } \\
\text { siempre }\end{array}$} \\
\hline & & fi & $\%$ & fi & $\%$ & $\mathbf{f i}$ & $\%$ \\
\hline \multirow{4}{*}{$\begin{array}{l}\text { Planeación por } \\
\text { resultados }\end{array}$} & $\begin{array}{l}\text { Participan los directores de } \\
\text { las IE en la elaboración del } \\
\text { plan estratégico de la UGEL }\end{array}$ & 12 & $40.0 \%$ & 15 & $\mathbf{5 0 . 0 \%}$ & 3 & $10.0 \%$ \\
\hline & $\begin{array}{l}\text { Los directores de las IE } \\
\text { conocen la visión y los } \\
\text { objetivos estratégicos de la } \\
\text { UGEL }\end{array}$ & 16 & $\mathbf{5 3 . 3 \%}$ & 13 & $43.3 \%$ & 2 & $6.7 \%$ \\
\hline & $\begin{array}{l}\text { Las metas educativas de las } \\
\text { IE están alineadas a las metas } \\
\text { planteadas en el PE de la } \\
\text { UGEL }\end{array}$ & 15 & $\mathbf{5 0 . 0 \%}$ & 13 & $43.3 \%$ & 1 & $3.3 \%$ \\
\hline & $\begin{array}{l}\text { La UGEL difunde directivas } \\
\text { y/o procedimientos para la } \\
\text { elaboración del plan anual de } \\
\text { las I.E. }\end{array}$ & 16 & $\mathbf{5 3 . 3 \%}$ & 13 & $43.3 \%$ & 1 & $3.3 \%$ \\
\hline \multirow{2}{*}{$\begin{array}{l}\text { Presupuesto } \\
\text { por resultados }\end{array}$} & $\begin{array}{l}\text { El presupuesto que destina la } \\
\text { UGEL para el desarrollo de } \\
\text { actividades de las I.E. se } \\
\text { relacionan con las metas. }\end{array}$ & 5 & $16.7 \%$ & 13 & $43.3 \%$ & 12 & $40.0 \%$ \\
\hline & $\begin{array}{l}\text { El presupuesto destinado } \\
\text { permite satisfacer las } \\
\text { necesidades de las I.E. }\end{array}$ & 13 & $43.3 \%$ & 7 & $23.3 \%$ & 10 & $33.3 \%$ \\
\hline
\end{tabular}




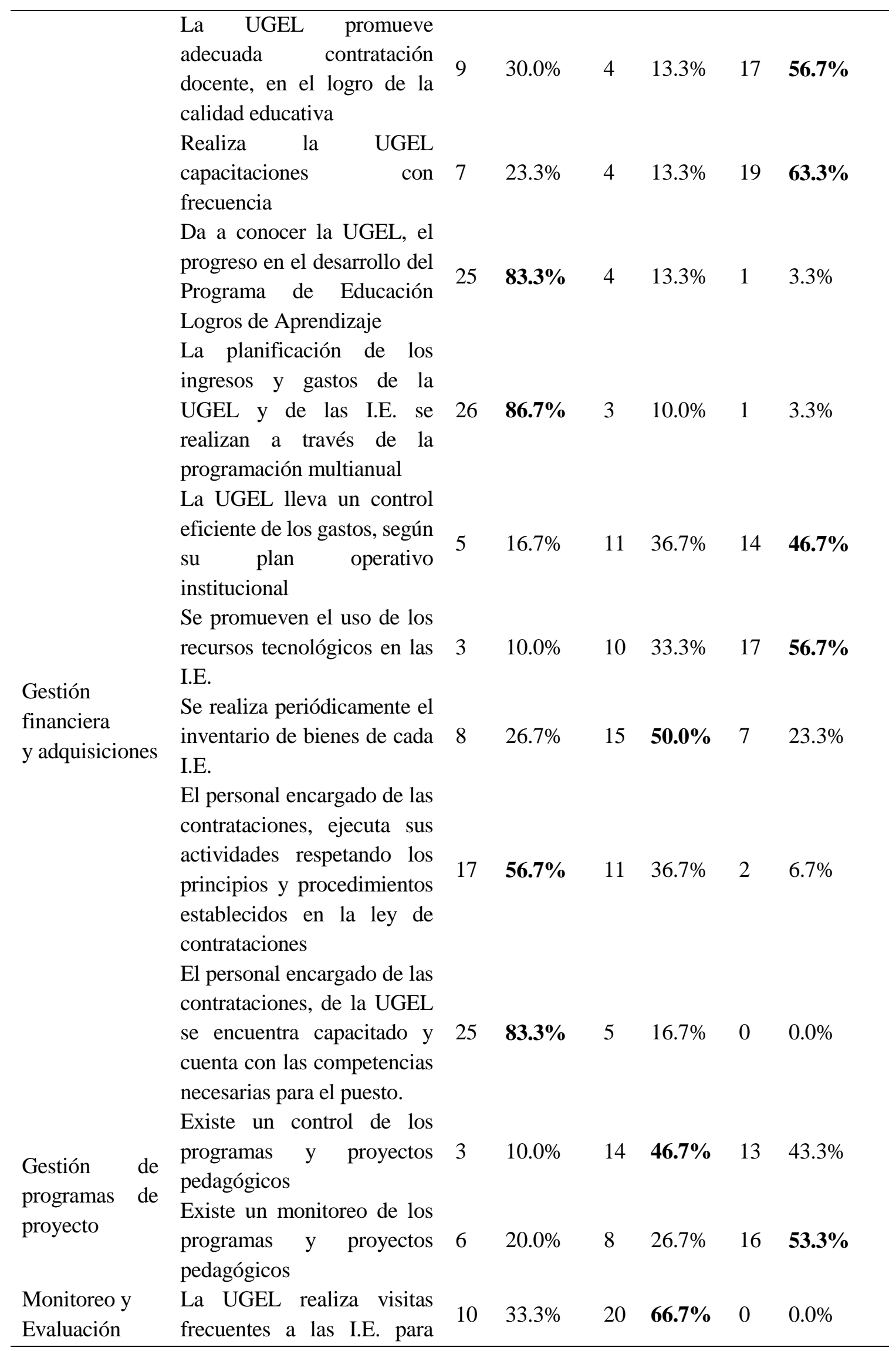


verificar el cumplimiento de

las actividades

La UGEL difunde sobre los

$\begin{array}{llllllll}\text { resultados alcanzados de } & 14 & 46.7 \% & 13 & 43.3 \% & 3 & 10.0 \%\end{array}$

acuerdo a su plan estratégico

Fuente: Cuestionario aplicado a los especialistas de la UGEL

Del $100 \%$ (30) de los encuestados, refiere a la dimensión planeación por resultados, el $53.3 \%$ (16) sostienen que "Nunca o casi nunca" los directores de las IE conocen la visión y los objetivos estratégicos de la UGEL, ni que la UGEL difunde directivas y procedimientos de manera oportuna para la elaboración de los planes institucionales. Y, que apenas las metas educativas de las I.E se encuentren alineadas a las metas planteadas en el plan estratégico de la UGEL (50\%) En cuanto, a la dimensión presupuesto por resultados, el 83.3\%(25) perciben que "Nunca o casi nunca", la UGEL, da a conocer el progreso del programa de educación logros de aprendizaje, y difícilmente, el presupuesto destinado permite satisfacer las necesidades de las instituciones (43.3\%). Sin embargo, el 63.3\% (19) aluden que "Siempre o casi siempre" la UGEL efectúa capacitaciones de manera continua, y promueve una adecuada contratación docente, deseando lograr una buena calidad educativa (56.7\%). En tanto, el 43.3\% (13), señalaron que "A veces" el presupuesto que destina la UGEL, para el desarrollo de actividades de las I.E se relacionan con las metas. Con respecto a la dimensión gestión financiera y adquisiciones, el $86.7 \%$ (26) opinan que "Nunca o casi nunca" la planificación de los ingresos y gastos se efectúan a través de un carácter multianual, ni el personal encargado de las contrataciones, de la UGEL, se encuentra capacitado y cuenta con las competencias para su cargo (83.3), tampoco se efectúan actividades respetando los principios y procedimientos establecidos en la ley de contrataciones (56.7\%). Asimismo, indican que, se realiza de manera periódica de los inventarios en las instituciones educativas (50\%). Por lo contrario, el 56.7(17) aseguran que "Siempre o casi siempre" se promueven el uso de los recursos tecnológicos en las I.E. y que la UGEL lleva un control eficiente de los gastos, según su POI. (46.7\%). En cuanto, a la dimensión gestión de programas de proyecto, el 53.3\% (16) señalan que "Siempre o casi siempre" existe un monitoreo de los programas y proyectos pedagógicos. Sin embargo, el $46.7 \%$ sostienen que "A veces" se efectúan los controles de dichos programas y proyectos. Por último, en la dimensión monitoreo y evaluación, el 66.7\%(20), sostienen que "A veces”, la UGEL efectúa visitas a las instituciones educativas. En el caso 
de las actividades de difusión de los resultados alcanzados por parte de la UGEL, estos se desarrollan "Nunca o casi nunca", según el 46.7\%(14) de los especialistas.

\section{Tabla 4}

Gestión por resultados desde la percepción de los directores de las I.E

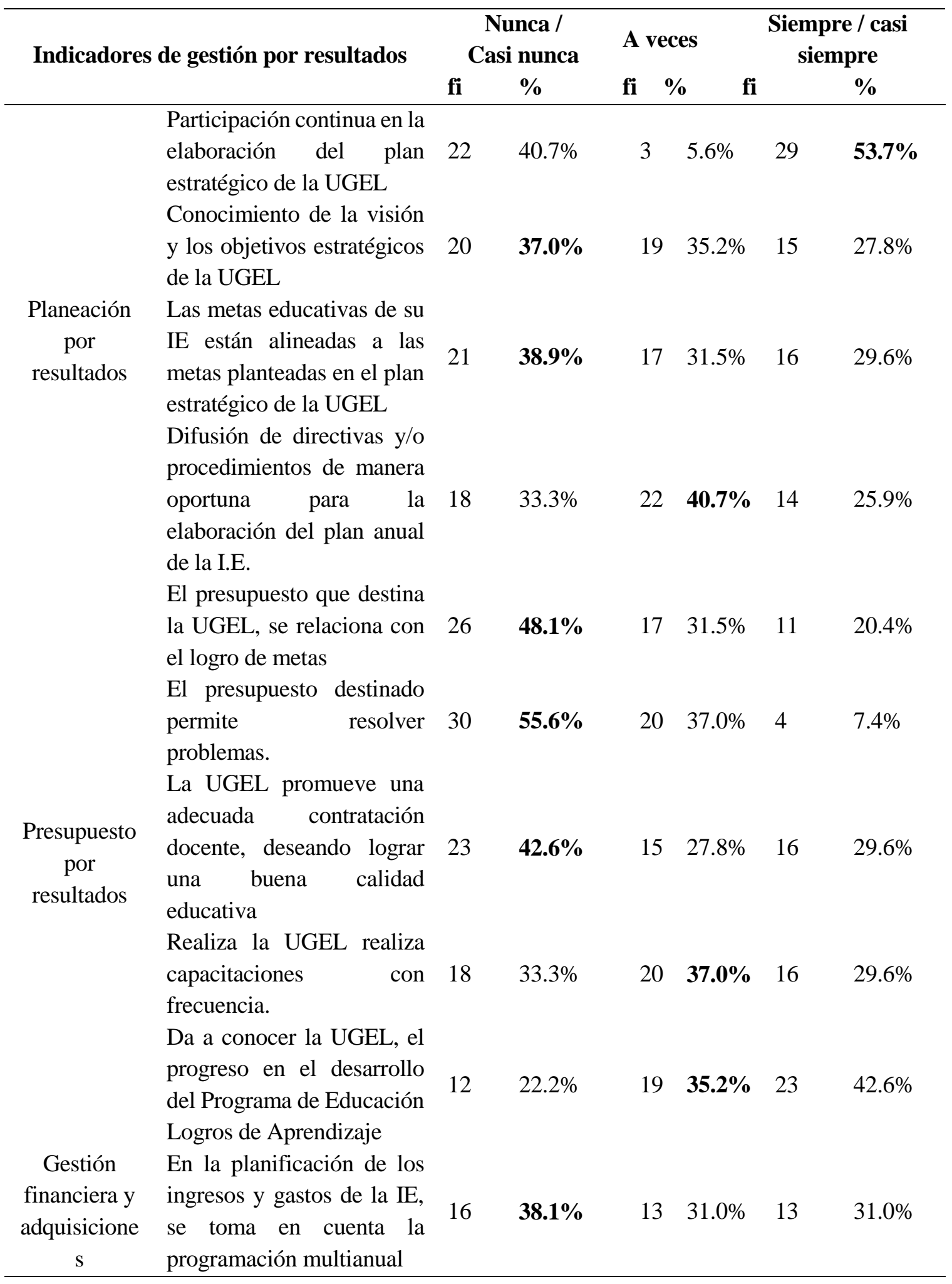




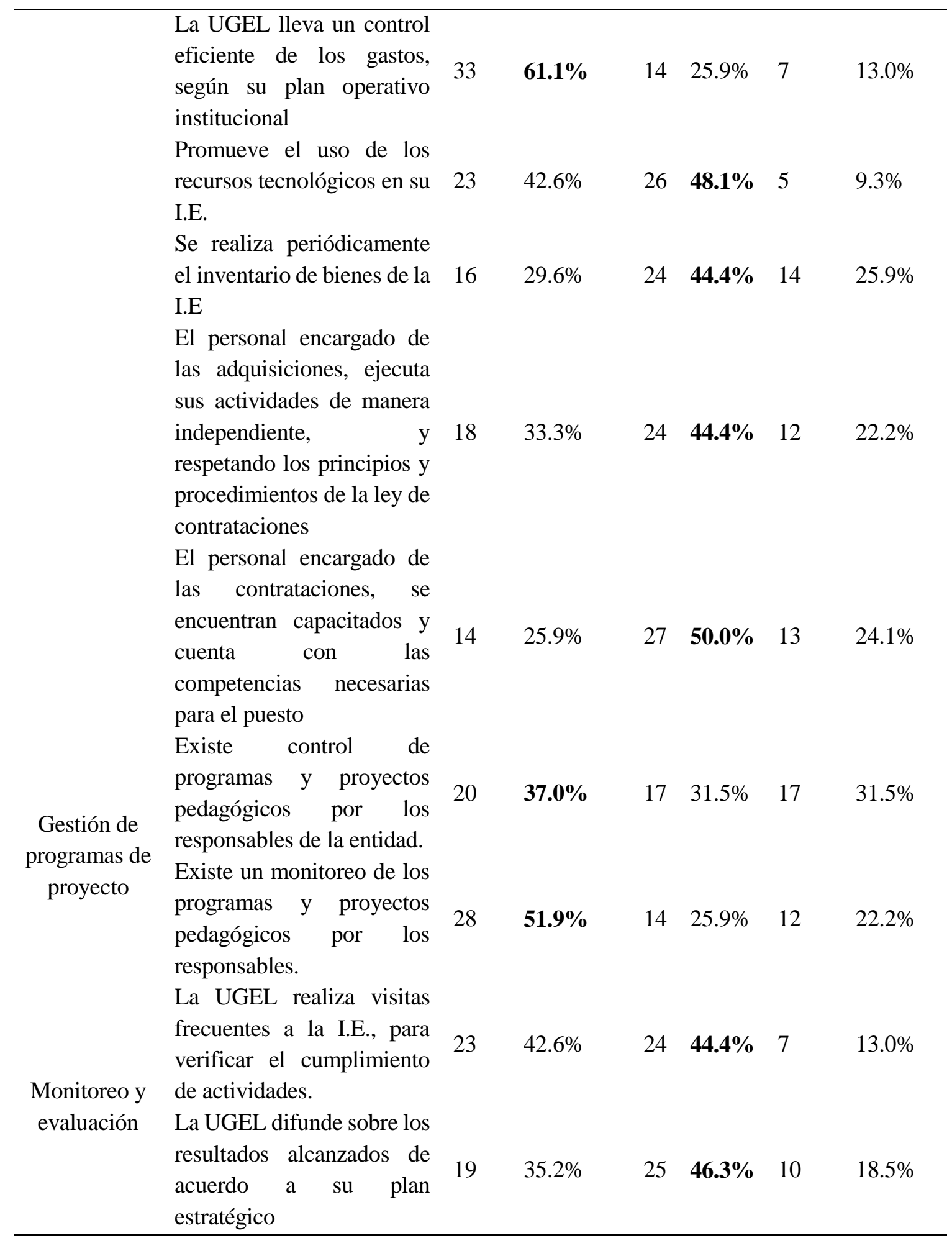

Fuente: Cuestionario aplicado a los directores de las I.E.

Del 100\% (54) de los directores de las instituciones educativas, Por lo que refiere a la planeación por resultados, el $53.7 \%$ (29) señalan que participan de manera continua en la elaboración del plan estratégico de la UGEL, y el 40.7\% (22) aluden que "A veces" la UGEL difunde las directivas y procedimiento de manera oportuna. Por lo contrario, el 
38.9\% sostienen que "Nunca o casi nunca" las metas educativas de las I.E están alineadas a las metas planteadas en el plan estratégico de la UGEL, ni conocen la visión y los objetivos estratégicos de la UGEL 37\%. En cuanto, al presupuesto por resultados, el $55.6 \%$ (30) aluden que el presupuesto recibido "Nunca o casi nunca" les permite resolver en su totalidad, los problemas de la institución, asimismo el presupuesto destinado por la UGEL, se relacionan con sus metas institucionales (48.1\%), ni promueve una adecuada contratación a fin de lograr una buena calidad educativa (42.6\%). Y "A veces", la UGEL realiza capacitaciones con frecuencia (37.2\%), y, da conocer el progreso en el desarrollo del programa de educación logros de aprendizaje (35.2\%). Con respecto a la gestión financiera y adquisiciones, el 61.1\% (33) señalan que "Nunca o casi nunca" la UGEL, lleva un control eficiente de los gastos, tampoco consideran la programación multianual en la planificación de los ingresos y gastos (38.1\%). Pero, “A veces”, el personal encargado de las contrataciones se encuentra capacitado (50\%), y que estos respetan los principios y procedimientos de la ley de contrataciones (44\%), periódicamente se realiza el inventario de bienes de las I.E. (44.4\%), y promueven el uso de los recursos tecnológicos en sus instituciones (48.1\%). En cuanto, a la gestión de programas de proyecto, el $51.9 \%$ y $37 \%$ señalan que "Nunca o casi nunca" existe un monitoreo y control de los programas y proyectos pedagógicos por parte de la UGEL respectivamente. Por último, en el monitoreo y evaluación, el 46.3\%(25), sostienen que "A veces", la UGEL difunde los resultados alcanzados de acuerdo al plan estratégico de su institución, y realiza visitas frecuentes a las I.E. a fin de verificar el cumplimiento de las actividades (44.4\%). Es así que el diagnóstico que ha desarrollado ha conllevado a presentar un modelo de gestión, el mismo que plantea el uso de manera integrado las diversas herramientas y métodos de carácter institucional,

\section{CONCLUSIÓN O CONSIDERACIONES FINALES}

El diagnóstico que se ha desarrollado dentro del contexto de estudio, muestra claras evidencias de procesos deficientes en la ejecución de gasto, estos no solo en la percepción de los diversos especialistas, sino también en los que conforman dichas unidades como son los directores, entonces el desarrollo de un modelo de gestión contribuye significativamente para la mejora de acciones, procesos o elementos de cambio, además de que cuenta con criterios apropiados para su validación. 


\section{LISTA DE REFERENCIAS}

Abanto, W. (2014). Diseño y desarrollo del proyecto de investigación: Guía del aprendizaje. ( $4^{\circ}$ ed.). Perú: Universidad Cesar Trujillo.

Álvarez, M. \& Polo, C. (2018). El coste del déficit público en España: el aumento en las tasas impositivas y la reducción del gasto público. Revista El trimestre económico, $85(340)$,

833-858.

https://www.redalyc.org/jatsRepo/313/31359237006/html/index.html

Arbaiza, L. (2019). Como elaborar una tesis de grado. Colombia: Esan Ediciones

Barrenechea, J (2018). Gestión por resultados y administración de la Unidad Ejecutora N001: MININTER, Lima, periodo 2017. Universidad Cesar Vallejo. Lima, Perú. Recuperado

de: https://www.mininter.gob.pe/sites/default/files/Eval\%20POI\%202017\%20VB\%20 $\underline{01 . p d f}$

Bastidas, C. y Andocilla, J. (2016). Criterios que se deben considerar para asegurar la efectividad de la calidad del gasto. 3(7). Recuperado de: https://revistapublicando.org/revista/index.php/crv/article/view/284

Bermeo, H; González, D; Hernández, I; \& Calderón, M. (2018). Citizen participation in the design of public policy through ICTs in Colombia. Revista Cuadernos de Administración, 34(60), 3-17. Recuperado de: https://www.redalyc.org/articulo.oa?id=225057030002

Bernal, C. (2010). Metodología de la investigación. ( $3^{\circ}$ ed.) Colombia: Pearson Educación.

Botero, J. \& García, J. (2018). Desarrollo, reestructuración del gasto público y alianzas público-privadas. Revista de Economía Institucional, 20(38), 185-2017. DOI: https://www.redalyc.org/jatsRepo/419/41956121008/index.html

Campo, J. \& Mendoza, H. (2018). Gasto público y crecimiento económico: un análisis regional para Colombia, 1984-2012. Revista Lecturas de Economía, 1(88), pp.77108. https://www.redalyc.org/jatsRepo/1552/155254736003/155254736003.pdf

Cantillo, M. y Buitrago, A. (2018). Nuevas miradas y enfoques de diversas investigaciones. Tomo II. Chile: Universidad Santiago de Cali.

CONCYTEC (2018). Compendio de normas para trabajos escritos. Colombia: Instituto Colombiano de Normas Técnicas y Certificación, CONCYTEC 
Chica, S. (2017). Análisis de la gestión por resultados en el desarrollo en perspectiva de buena gobernanza y el cumplimiento de las metas concernientes al desarrollo sostenible en Colombia 14(27), 25-60. Colombia. Recuperado de: http://www2.congreso.gob.pe/sicr/cendocbib/con5_uibd.nsf/A2D9B3A71F7D916 E0525824B006FACEC/\$FILE/chicaser.pdf

Dávila, L. (2019). Gestión por resultados y ejecución presupuestaria de la Municipalidad Distrital de la Banda de Shilcayo, año 2018. Universidad Cesar Vallejo, Perú. Recuperado

de: http://repositorio.ucv.edu.pe/bitstream/handle/UCV/34707/D\%C3\%A1vila_PLJ.p df? sequence $=1 \&$ isAllowed $=y$

Ferrer, E. (2018) Strategic project management: a methodology for sustainable competitive advantage. Revista EAN, 56(5), 15-31. DOI: https://www.redalyc.org/articulo.oa?id=20657725002

Fonseca, M. \& López, E. (2018). Características generales del recaudo y gasto público del Estado de Cundinamarca, 1856-1885. Revista tiempo \& economia, 5(1), 1-17. Recopilado de: https://www.redalyc.org/jatsRepo/5745/574562191003/index.html

Franciskovic, J. (2016). Desafíos de la gestión pública: Elaboración del Presupuesto por resultados. Revista ESAN, 5(56), 56-87.

Galiya, A; Malika, K; Kuanyshbek, A; Salima, S; \& Gulmira, B (2018). Managerial competence of future specialists of the education system. Preschool education and upbringing and medicine in the comparative aspect. Revista opción. 34(85), 44-62. Recuperado de: https://www.redalyc.org/articulo.oa?id=31055914002

García, J. (2015). Gestión pública y valor público. (Artículo científico). Revista Actualidad Gubernamental. 18(5), 56-87.

Gormas, E. (2019). Evaluación del presupuesto por resultados y la calidad de gasto en el Proyecto Especial Huallaga Central y Bajo Mayo, año 2018. Universidad Cesar Vallejo. Recuperado de: http://repositorio.ucv.edu.pe/bitstream/handle/UCV/31895/Gormas_JEG.pdf?sequ ence $=1 \&$ isAllowed $=\mathrm{y}$

Levy, N. (2016). Política fiscal y desequilibrios económicos: el impacto de la composición del gasto público sobre el crecimiento de la economía mexicana (Artículo 
científico). Revista Economíaunam, 13(39), 82-105. DOI: https://www.redalyc.org/pdf/3635/363546801004.pdf

Medina, A. y Hernández, A. (2017). Análisis de los procedimientos de gestión por resultados. Ingeniare. Revista chilena de ingeniería, 27(2), 35-46. Recuperado de: https://scielo.conicyt.cl/scielo.php?pid=S071833052019000200328\&script=sci_arttext\&tlng=p

Ministerio de Economía y Finanzas (2016). Procedimientos asignados en los programas de presupuesto por resultados. Lima, Perú. Recuperado de: https://www.mef.gob.pe/es/presupuesto-por-resultados.

Ramos, J. (2016) Gestión por resultados en México, 2013-2014. Algunos impactos en Baja California. Revista Estudios Fronterizos. 17(34), 64-84. DOI: https://www.redalyc.org/articulo.oa?id=53046485004_2

Ríos, W. (2018). Evaluación de la gestión por resultados y calidad de gasto en la Policía Nacional del Perú (Artículo científico). Universidad Cesar Vallejo. Lima, Perú. Recuperado

de: http://repositorio.ucv.edu.pe/bitstream/handle/UCV/21654/Rios_AW.pdf?sequenc $\mathrm{e}=1 \&$ is Allowed $=\mathrm{y}$

Silva, G. \& Mejía, F. (2017). Estados presupuestarios. (4ed). Perú: A Empresarial

Soberanes, M; Gómez, R; \& Andrade, M. (2018) Opportunity Strategies related to Business Competitiveness in Restaurants in Mexico. Case: Micro-enterprises in the Municipality of Othon P. Blanco, State of Quintana Roo. (Artículo científico). Revista de cuadernos de administración. 34(60), 18-29. DOI: https://www.redalyc.org/articulo.oa?id=225057030003

Torres, R. (2017). "Participación Ciudadana y Educación Una Mirada Amplia y 20 Experiencias en América Latina". ( $\left.3^{\circ} \mathrm{ed}\right)$. Colombia: Paraninfo

UNESCO (2017). Educación para el siglo XXI. Organización de las Naciones Unidad para la educación, la Ciencia y la Cultura. Recuperado de: https://goo.gl/CzftAY

Vargas, S. (2018). Evaluación de las acciones administrativas y la gestión por resultados en la Municipalidad Provincial de San Martín, año 2018. Universidad César Vallejo, Perú. Recuperado de: http://repositorio.ucv.edu.pe/bitstream/handle/UCV/28750/Vargas_OS.pdf?sequen ce $=1 \&$ is Allowed $=y$ 